\title{
MINIMAL PERIODIC ORBITS AND TOPOLOGICAL ENTROPY OF INTERVAL MAPS
}

\author{
BAU-SEN DU
}

\begin{abstract}
For any two integers $m \geq 0$ and $n \geq 1$, we construct continuous functions from $[0,1]$ into itself which have exactly one minimal periodic orbit of least period $2^{m}(2 n+1)$, but with topological entropy equal to $\infty$.
\end{abstract}

Introduction. Let $I$ denote the unit interval $[0,1]$ and let $g \in C^{0}(I, I)$. If $g$ has a periodic point of least period $2^{m}(2 n+1)$, where $m \geq 0$ and $n \geq 1$ are integers, then it is well known [3] that the topological entropy of $g$ is greater than or equal to $\left(\log \lambda_{n}\right) / 2^{m}$, where $\lambda_{n}$ is the (unique) positive zero of the polynomial $x^{2 n+1}-2 x^{2 n-1}-1$. The converse is false, but known counterexamples are rather complicated [4, p. 407]. The purpose of this note is to indicate how to use an easy and well-known method to construct examples which are simpler but with stronger properties than those given in [4, p. 407] except that our examples are not piecewise monotone. As a consequence of our construction, we also obtain the well-known example $g_{\infty}$ as described in [8, p. 14] which has exactly one periodic orbit of least period $2^{m}$ for every $m \geq 0$ and no other periodic orbits. It is worth mentioning that the set of all periodic points of the example $g_{\infty}$ described in $[8$, p. 14] is not closed. This is in contrast to the fact [7] that if the set of all periodic points of a continuous function in $C^{0}(I, I)$ is closed, then this function can only have periodic points of periods some powers of 2 .

The construction. For every continuous function $g$ in $C^{0}(I, I)$, let $G:[0,3] \rightarrow$ $[0,3]$ be the continuous function defined by (i) $G(x)=g(x)+2$ for $0 \leq x \leq 1$; (ii) $G(x)=x-2$ for $2 \leq x \leq 3$; and (iii) $G$ is linear on $[1,2]$. Then it is clear that $G^{2} \mid I=g$. Now let $\tilde{g}$ be the scaled-down copy of $G$ on $I$. That is, $\tilde{g}(x)=[g(3 x)+2] / 3$ for $0 \leq x \leq 1 / 3 ; \tilde{g}(x)=[2+g(1)](2 / 3-x)$ for $1 / 3 \leq x \leq 2 / 3$; and $\tilde{g}(x)=x-2 / 3$ for $2 / 3 \leq x \leq 1$. It follows from [1] that the topological entropy of $\tilde{g}$ is greater than or equal to one half of that of $g$. This function $\tilde{g}$ is called the renormalized square root of $g$ on $I$. For every continuous function $g_{0}$ in $C^{0}(I, I)$, we define the sequence $\left\langle g_{m}\right\rangle(m \geq 1)$ inductively by letting $g_{m}$ be the renormalized square root of $g_{m-1}$ on $I$. This sequence $\left\langle g_{m}\right\rangle(m \geq 1)$ is called the sequence of successive renormalized square roots of $g_{0}$ on $I$.

For every positive integer $k$, choose $2 k+2$ real numbers $a_{k, i}$ with $0=a_{k, 0}<$ $a_{k, 1}<a_{k, 2}<\cdots<a_{k, 2 k+1}=1$. Let $p_{k}$ be the continuous function in $C^{0}(I, I)$ defined by (i) $p_{k}\left(a_{k, i}\right)=0$ for all even $i$; (ii) $p_{k}\left(a_{k, i}\right)=1$ for all odd $i$; and (iii) $p_{k}$ is linear on each interval $\left[a_{k, i}, a_{k, i+1}\right], 0 \leq i \leq 2 k$. Let $q_{k}$ be the continuous function

\footnotetext{
Received by the editors April 20, 1985.

1980 Mathematics Subject Classification (1985 Revision). Primary 26A18; Secondary 54C70, $58 \mathrm{~F} 20$.

Key words and phrases. Least period, periodic points, periodic orbits, minimal periodic orbits, topological entropy.
} 
from the interval $[1 /(k+1), 1 / k]$ onto itself which is the scaled-down copy of $p_{k}$ on $[1 /(k+1), 1 / k]$. That is,

$$
q_{k}(x)=1 /(k+1)+p_{k}(k(k+1)(x-1 /(k+1))) /[k(k+1)] .
$$

Finally, let $f_{0} \in C^{0}(I, I)$ be the continuous function defined by $f_{0}(0)=0$ and $f_{0}(x)=q_{k}(x)$ on $[1 /(k+1), 1 / k]$ for each positive integer $k$ and let $\left\langle f_{m}\right\rangle(m \geq 1)$ be the sequence of successive renormalized square roots of $f_{0}$ on $I$. Now we can state the following theorem whose proof is easy and omitted. (For the definition of minimal periodic orbits, see [2 or 5].)

THEOREM 1. Let the sequence $\left\langle f_{m}\right\rangle(m \geq 0)$ be defined as above. Then $\left\langle f_{m}\right\rangle$ is a uniformly convergent sequence in $C^{0}(I, I)$ with the following two properties:

(1) For every integer $m \geq 0, f_{m}$ has infinitely many minimal periodic orbits of least period $2^{m} \cdot 3$ and the topological entropy of $f_{m}$ is $\infty$.

(2) If $f$ is the uniform limit of the sequence $\left\langle f_{m}\right\rangle$, then $f$ is exactly the same as the function $g_{\infty}$ described in $[\mathbf{8}, p .14]$ with zero topological entropy [6].

In the above theorem, every function $f_{k}$ has minimal periodic orbits of least period $2^{k} \cdot 3$. In the following, we will use these functions $f_{k}$ to construct, for any two integers $m \geq 0$ and $n \geq 1$, continuous functions $F_{m, n}$ in $C^{0}(I, I)$ which have exactly one minimal periodic orbit of least period $2^{m}(2 n+1)$, but with topological entropy equal to $\infty$.

For every positive integer $n$, let $u_{n}$ be any continuous function from $[2 / 3,1]$ into itself with exactly one minimal periodic orbit [9] (see [2, 5] also) of least period $2 n+1$ and let $F_{0, n}$ be the continuous function in $C^{0}(I, I)$ defined by (i) $F_{0, n}(x)=f_{k}(3 x) / 3$ for $0 \leq x \leq 1 / 3$, where $k$ is any positive integer and $f_{k}$ is defined as in Theorem 1 ; (ii) $F_{0, n}(x)=u_{n}(x)$ for $2 / 3 \leq x \leq 1$; and (iii) $F_{0, n}$ is linear on $[1 / 3,2 / 3]$. It is clear that $F_{0, n}$ has exactly one minimal periodic orbit of least period $2 n+1$ and its topological entropy is $\infty$. For any fixed integer $n>0$, let $\left\langle F_{m, n}\right\rangle(m \geq 1)$ be the sequence of successive renormalized square roots of $F_{0, n}$ on $I$.

Now we can state the following theorem whose proof is again easy and omitted.

THEOREM 2. For every positive integer $n$, let the sequence $\left\langle F_{m, n}\right\rangle(m \geq 0)$ be defined as above. Then for every fixed $n>0,\left\langle F_{m, n}\right\rangle(m \geq 0)$ is a uniformly convergent sequence in $C^{0}(I, I)$ with the following two properties:

(1) For every integer $m \geq 0$, the function $F_{m, n}$ has exactly one minimal periodic orbit of least period $2^{m}(2 n+1)$ and the topological entropy of $F_{m, n}$ is $\infty$.

(2) If $F_{n}$ is the uniform limit of the sequence $\left\langle F_{m, n}\right\rangle$, then $F_{n}=f$, where $f$ is defined as in Theorem 1.

REMARK. We can also construct functions $G_{m, n}$ in $C^{0}(I, I)$ with the properties as stated in part (1) of Theorem 2 as follows: For any two integers $m \geq 0$ and $n \geq 1$, let $v_{m, n}$ be any continuous function from $[2 / 3,1]$ into itself which has exactly one minimal periodic orbit $[\mathbf{2}, \mathbf{5}]$ of least period $2^{m}(2 n+1)$. Let $G_{m, n}$ be the continuous function in $C^{0}(I, I)$ defined by (i) $G_{m, n}(x)=f_{k}(3 x) / 3$ for $0 \leq x \leq 1 / 3$, where $k>m$ is any integer and $f_{k}$ is defined as in Theorem 1 ; (ii) $G_{m, n}(x)=v_{m, n}(x)$ for $2 / 3 \leq x \leq 1$; and (iii) $G_{m, n}$ is linear on [1/3,2/3]. Then it is easy to see that $G_{m, n}$ has exactly one minimal periodic orbit of least period $2^{m}(2 n+1)$ and the topological entropy of $G_{m, n}$ is $\infty$. 


\section{REFERENCES}

1. R. Adler, A. Konheim, and M. McAndrew, Topological entropy, Trans. Amer. Math. Soc. 114 (1965), 309-319.

2. L. Alseda, J. Llibre, and R. Serra, Minimal periodic orbits for continuous maps of the interval, Trans. Amer. Math. Soc. 286 (1984), 595-627.

3. L. Block, J. Guckenheimer, M. Misiurewicz, and L.-S. Young, Periodic points and topological entropy of one dimensional maps, Lecture Notes in Math., vol. 819, Springer-Verlag, Berlin and New York, 1980, pp. 18-34.

4. W. A. Coppel, Sarkovskii-minimal orbits, Math. Proc. Cambridge Philos. Soc. 93 (1983), 397-408.

5. C.-W. Ho, On the structure of the minimum orbits of periodic points for maps of the real line (to appear).

6. M. Misiurewicz, Horseshoes for mappings of the interval, Bull. Acad. Polon. Sci. 27 (1979), 167-169.

7. Z. Nitecki, Maps of the interval with closed periodic set, Proc. Amer. Math. Soc. 85 (1982), 451-456.

8. __ Topological dynamics on the interval, Progress in Math., vol. 21, Birkhäuser, Boston, Mass., 1982, pp. 1-73.

9. P. Stefan, $A$ theorem of Sharkovsky on the existence of periodic orbits of continuous endomorphisms of the real line, Comm. Math. Phys. 54 (1977), 237-248.

Institute of Mathematics, ACademia Sinica, Taipei, Taiwan 115, Republic of 